МИНИСТЕРСТВО ОБРАЗОВАНИЯ И НАУКИ РОССИЙСКОЙ ФЕДЕРАЦИИ

ТОМСКИЙ ГОСУДАРСТВЕННЫЙ УНИВЕРСИТЕТ

\title{
МАТЕРИАЛЫ
}

VIII Международной молодежной научной конференции

«МАТЕМАТИЧЕСКОЕ

И ПРОГРАММНОЕ ОБЕСПЕЧЕНИЕ

ИНФОРМАЦИОННЫХ, ТЕХНИЧЕСКИХ

И ЭКОНОМИЧЕСКИХ СИСТЕМ»

Томск, 26-30 мая 2021 г.

Под общей редакиией И.С. Шмырина

Томск

Издательство Томского государственного университета 2021 
стои, вызванные временем поиска луча, которое составляет порядка нескольких миллисекунд.

\title{
ЛИТЕРАТУРА
}

1. David K., Berndt H. 6G vision and requirements: Is there any need for beyond 5G? // IEEE Vehicular Technology Magazine. - 2018. - V. 13. - No. 3. - P. 72-80.

2. Petrov V., Kurner T., Hosako I. IEEE 802.15. 3d: First standardization efforts for sub-terahertz band communications toward 6G // IEEE Communications Magazine. - 2020. - V. 58. - No. 11. - P. 28-33.

3. Tousi $Y$., Afshari E. A high-power and scalable 2-D phased array for terahertz cmos integrated systems // IEEE Journal of Solid-State Circuits. - 2014. - V. 50. - No. 2. - P. 597-609.

4. Petrov V., Komarov M., Moltchanov D., Jornet J.M., Koucheryavy Y. Interference and sinr in millimeter wave and terahertz communication systems with blocking and directional antennas // IEEE Transactions on Wireless Communications. - 2017. - V. 16. - No. 3. - P. 1791-1808.

5. Petrov V., Moltchanov D., Koucheryavy Y., Jornet J.M. The effect of small-scale mobility on terahertz band communications // Proceedings of the 5th ACM International Conference on Nanoscale Computing and Communication. 2018. - P. $1-2$.

6. Petrov V., Moltchanov D., Koucheryavy Y., Jornet J.M. Capacity and outage of terahertz communications with user micro-mobility and beam misalignment // IEEE Transactions on Vehicular Technology. - 2020.

7. 3GPP, "NR; Multi-connectivity; stage 2 (Release 16),"3GPP TS 37.340V16.0.0, December 2019.

8. Begishev V., Sopin E., Moltchanov D., Pirmagomedov R., Samuylov A., Andreev S., Koucherayvy Y., Samouylov K. Performance Analysis of Multi-Band Microwave and Millimeter-Wave Operation in 5G NR Systems // IEEE Transactions on Wireless Communications. - 2021.

9. Shafie A., Yang N., Han C. Multi-connectivity for indoor terahertz communication with self and dynamic blockage // ICC 2020-2020 IEEE International Conference on Communications (ICC). - 2020. - P. 1-7.

10. Moltchanov D. Distance distributions in random networks // Elsevier Ad Hoc Networks. - August 2012. V. 10. - P. 1146-1166.

11. Begishev V., Moltchanov D., Sopin E., Samuylov A., Andreev S., Koucheryavy Y., Samouylov K. Quantifying the impact of guard capacity on session continuity in 3GPP new radio systems // IEEE Transactions on Vehicular Tech. 2019. - V. 68. - No. 12. - P. 12345-12359.

12. Kurner T., Fricke A., Rey S., Le Bars P., Mounir A., Kleine-Ostmann T. Measurements and modeling of basic propagation characteristics for intra-device communications at $60 \mathrm{GHz}$ and $300 \mathrm{GHz} / /$ Journal of Infrared, Millimeter, and Terahertz. - 2015. - V. 36. - No. 2. - P. 144-158.

13. Mokrov E., Ponomarenko-Timofeev A., Gudkova I., Masek P., Hosek J., Andreev S., Koucheryavy Y., Gaidamaka $Y$. Modeling transmit power reduction for a typical cell with licensed shared access capabilities // IEEE Vehicular Technology Magazine. - 2018. - V. 67. - No. 6. - P. 5505-5509.

14. Constantine A.B. et al. Antenna theory: analysis and design. - Microstrip Antennas, John Wiley \& Sons, 2005.

15. IEEE, "Telecommunications and information exchange between systems local and metropolitan area networks - specific requirements. Part 11. Amendment 3," IEEE standard for information technology, 2012.

16. Cinlar E. Markov renewal theory // Advances in Applied Probability. - 1969. - V. 1. - No. 2. - P. 123-187.

DOI: $10.17223 / 978-5-907442-42-9-2021-20$

\section{МОДЕЛЬ ПРИОРИТЕТНОГО ДОСТУПА ТРАФИКА URLLC С АДАПТИВНЫМ ИЗМЕНЕНИЕМ СКОРОСТИ ТРАФИКА ЕМВВ В СЕТИ 5G: ЗАДАЧА ОПТИМИЗАЦИИ}

\author{
Кущазли А.И., Агеева А.С., Харин П.А. \\ Российский университет дружбы народов \\ aikushch@yandex.ru, anastasia.ageeva.it@gmail.com, gruzavjeg@mail.ru
}

\section{Введение}

На сегодняшний день в цифровом обществе с каждым днём растет роль телекоммуникационных услуг. Мы уже не можем представить себе жизнь без скоростного интернета, улучшенной сотовой сети. В современных быстро развивающихся телекоммуникационных технологиях услуги мобильной связи широко проникают во все слои жизни общества, тем самым беспроводная сеть 5-го поколения покроет более широкий диапазон использования. Услуги 5G поставляются с различными вариантами использования, такими как расширенная мобильная широкополосная связь (еМВВ), сверхнадежная связь с низкой задержкой (URLLC) и массовая связь машинного типа (mMTC). Если же рассматривать каждую услугу по отдельности, то к отличительной особенности URLLC являются требования к задержке, требования же к еMBB это вы- 
сокая скорость передачи данных, a mMTC это возможность поддержки высокой плотности соединений $[1,2]$.

Стандарты сотовой сети растут вместе с требованиями промышленного сектора и обычных пользователей. Практически в каждом секторе бизнеса существует повышенный спрос на новые или усовершенствованные приложения, которые повышают производительность труда или улучшают качество обслуживания клиентов. Сочетание всех возможностей устройств с более быстрой, высокой пропускной способностью и высоко интеллектуальными сетями будет способствовать широкому экспериментированию и внедрению передовых мультимедийных приложений, которые способствуют и будут способствовать увеличению трафика и поддержки обеспечения работы новых сценариев использования в будущем.

В работе рассматривается модель совместного обслуживания трафика URLLC и eMBB в рамках одной базовой станции. Трафик первого типа считается приоритетным в силу своих высоких требований к задержке, а трафик второго типа имеет высокую скорость. Вследствие приоритета первый тип трафика преобладает над вторым типом трафика. В ситуации отсутствия свободных ресурсов для обслуживания URLLC трафика, выделенные ресурсы для еMBВ трафика будут уменьшаться вплоть до полного изъятия.

\section{1. Постановка задачи}

Совместная работа услуг URLLC и еMBB на одном радио-ресурсе приводит к возникающей при пользовании проблеме планирования, которую достаточно сложно решить ввиду требований к QoS и расположенного в основе компромисса между правилами и условиями работы этих услуг. Следует отметить, что в отличие от трафика URLLC, требования к еMBB менее восприимчивы с точки зрения задержки, и, следовательно, обслуживание может быть отложено в пользу обслуживания более приоритетного URLLC трафика.

Рассмотрим задачу исследования совместного обслуживания трафика URLLC и еMBB в рамках одной базовой станции. Выделяемые ресурсы представлены в виде ресурсных блоков PRB (Physical Recourse Block). Для обслуживания eMBB трафика выделяются слоты, которые в последствии будут называться ресурсными блоками, для URLLC выделяются мини-слоты, которые будут именоваться как ресурсные единицы.

Рассматривается одна сота с одной базовой станицей внутри, в области которой пользователи пытаются установить соединение. В ситуации отсутствия свободных ресурсов для обслуживания URLLC трафика, выделенные ресурсы для еMBВ трафика будут уменьшаться вплоть до полного изъятия [3].

Емкость системы представлена в виде $N$ ресурсных блоков, которые используются для обслуживания еМВВ трафика. В свою очередь, каждый ресурсный блок состоит из $b_{1}$ ресурсных единиц, которые используются для обслуживания URLLC трафика. Соответственно общее количество ресурсных единиц равно $C=N \cdot b_{1}$.

Предположим, что поступающая на обслуживание сессия URLLC занимает одну ресурсную единицу и общее число активных сессий URLLC в некоторый момент времени составляет $n$. Сессии типа еМВВ поступают на обслуживание на максимальной скорости, занимая целый ресурсный блок. При поступлении сессии URLLC скорость обслуживания сессии еМВВ может быть уменьшена ввиду приоритета сверхнадёжного трафика. Для обозначения числа активных сессий еMBВ с учетом их текущей скорости используется вектор $\vec{m}=\left(m_{1}, . ., m_{K}\right)$, в то время как вектор скоростей обслуживания сессий еМВВ $\vec{b}=\left(b_{1}, . ., b_{K}\right)$, при этом $b_{1}>b_{2}>\ldots>b_{K}$.

Состояния системы представлены следующим образом: $\left(m_{1}, \ldots, m_{K}, n\right)=(\vec{m}, n)=\vec{x}$. 
Сессии обоих типов трафика поступают в соответствии с пуассоновским законом и их интенсивности равны $\lambda_{m}$ и $\lambda_{u}$. Следует отметить, что индекс $m$ соответствует трафику еMBB, а $u$ - URLLC.

Предполагается, что время обслуживания сессий URLLC и еMBB распределено экспоненциально. Для трафика URLLC интенсивность равна $\mu_{u}$, а для еMBB - равна $\mu_{m}$ и не зависит от скорости обслуживания. Соответственно, нагрузка на систему будет иметь вид: $\rho_{m}=\frac{\lambda_{m}}{\mu_{m}}$ и $\rho_{u}=\frac{\lambda_{u}}{\mu_{u}}$ [4].

Схема модели со снижением и прерыванием обслуживания показана на рис. 1.

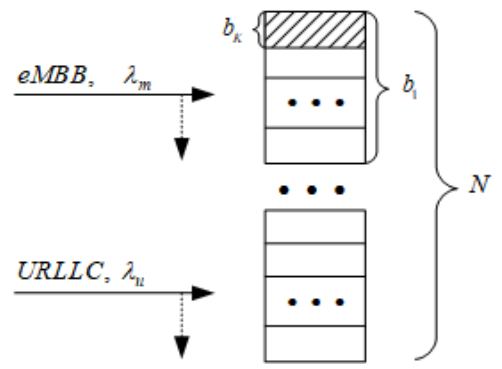

Рис. 1. Схема модели со снижением скорости и прерыванием обслуживания трафика еМВВ

Используя системную модель, опишем процесс совместного обслуживания трафика eMBB и URLLC. Если $C$ - максимальная загрузка системы, то пространство состояний выглядит следующим образом:

$$
N=\left\{\begin{array}{l}
(\vec{m}, n)=\left(m_{1}, \ldots, m_{k}, n\right): m_{k} \geq 0, \sum_{k=1}^{K} m_{k} \cdot b_{k} \leq C, \\
0 \leq n \leq C, \sum_{k=1}^{K} m_{k} \cdot b_{k}+n \leq C, k=\overline{1, K} .
\end{array}\right\}
$$

Опишем возможные ситуации для обоих видов трафика. Так при возникновении события с интенсивностью $\lambda_{m}$, т.е. поступает запрос на обслуживание еMBВ трафика:

- Если в системе есть хотя бы один свободный ресурсный блок, то новая сессия еМВВ принимается к обслуживанию.

- Если в системе нет свободных ресурсных блоков, то новая сессия еМВВ блокируется.

Возможные ситуации при возникновении события с интенсивностью $\lambda_{u}$, т.е. поступает запрос на обслуживание URLLC трафика:

- Если в системе есть хотя бы одна свободная ресурсная единица, то новая сессия URLLC принимается к обслуживанию.

- Если в системе нет свободной ресурсной единицы и в системе обслуживается хотя бы одна еМВВ сессия не на минимальной скорости, тогда скорость обслуживания еMBB сессии снижается на одну скорость и URLLC сессия поступает на обслуживание.

- Если в системе нет свободной ресурсной единицы и в системе обслуживается как минимум одна еМВВ сессия на минимальной скорости, то обслуживание еМВВ сессии прерывается и URLLC сессия поступает на обслуживание.

- Если в системе нет свободной ресурсной единицы и нет активной еМВВ сессии, то новая сессия URLLC блокируется.

Возможные ситуации при возникновении события с интенсивностью $\mu_{m}$, т.е. обслуживание еМВВ трафика окончено:

- Обслуживание сессии еМВВ трафика прекращается и происходит освобождение ресурсов. 
Возможные ситуации при возникновении события с интенсивностью $\mu_{u}$, т.е. обслуживание URLLC трафика окончено:

- Если в системе не обслуживаются сессии еМВВ или обслуживаются только на максимальной скорости, тогда обслуживание URLLC сессии прекращается и происходит освобождение ресурсов.

- Если в системе обслуживается как минимум одна еМВВ сессия не на максимальной скорости, тогда, когда обслуживание URLLC сессии прекращается, происходит освобождение ресурсов и скорость активной сессии еМВВ восстанавливается на единицу.

Диаграмма интенсивности переходов из центрального состояния представлена на рис. 2.

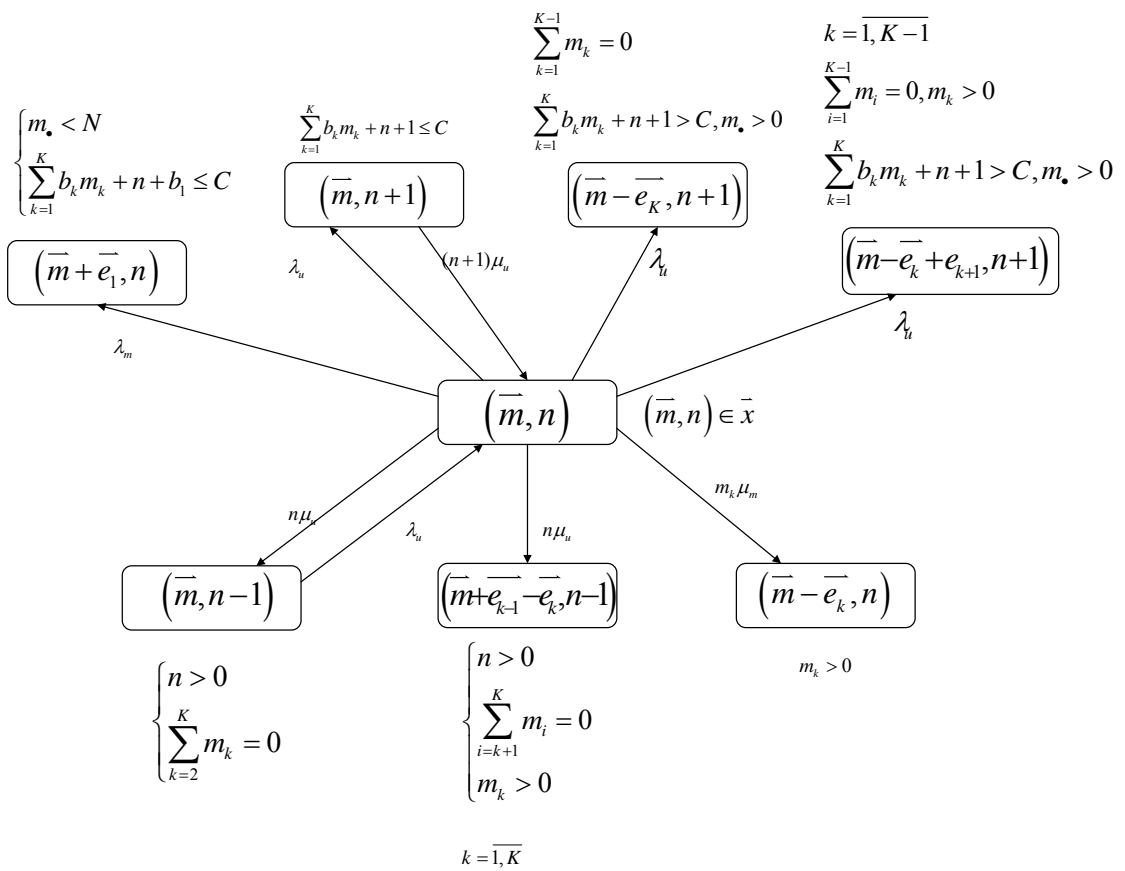

Рис. 2. Диаграмма интенсивности переходов

\section{2. Вероятностно-временные характеристики}

Рассматриваются следующие вероятностно-временными характеристики: среднее количество еMBB и URLLC сессий, вероятность блокировки запросов еMBB и URLLC сессий, средняя скорость сессий еМВВ.

- Среднее количество сессий еMBB $\left(\bar{m}_{k}\right)$ и $\operatorname{URLLC}(\bar{n})$ :

$$
\bar{m}_{k}=\sum_{m_{k}^{*}=1}^{N} m_{k} \cdot\left(\sum p(\bar{m}, n)\right), \bar{n}=\sum_{n=1}^{C}\left(n \cdot \sum_{\vec{m} \in \mathrm{N}} p(\vec{m}, n)\right) .
$$

- Вероятность блокировки запросов еMBB $\left(B_{m}\right)$ и $\operatorname{URLLC}\left(B_{u}\right)$ :

$$
B_{m}=\sum_{(\vec{m}, n) \in \mathrm{M}} p(\vec{m}, n),
$$

где $\mathrm{M}=(\vec{m}, n) \in \mathcal{N}: \sum_{i=1}^{K} m_{i} \cdot b_{i}+n+b_{1}>C, B_{u}=p(\overrightarrow{0}, C)$.

- Средняя скорость сессий еMBВ $(\bar{b})$ : 


$$
\bar{b}=\frac{1}{\sum_{\left(\mathcal{N} / \mathcal{N}_{0}\right)} p(\vec{m}, n)} \cdot \sum_{k=1}^{K} \sum_{(\vec{m}, n) \in \mathcal{N}_{k}} b_{k} \cdot p(\vec{m}, n),
$$

где $\mathcal{N}_{k}=(\vec{m}, n) \in \mathcal{N}: \sum_{i=1}^{k-1} m_{i}=0, m_{k}>0, k=\overline{1, K}$.

Пространство состояний $\mathcal{N}$ разбивается на $k$ непересекающихся множеств, образуя множества $\mathcal{N}_{k}$, т.е. $\mathcal{N}=\bigcup_{k=0}^{K} \mathcal{N}_{k}$.

При расчете средней скорости еМВВ заявок стоит рассматривать только те состояния, в которых присутствует как минимум одна активная еМВВ сессия. Следовательно, не учитываются состояния, в которых отсутствуют еМВВ заявки $\left(\mathcal{N} / \mathcal{N}_{0}\right)$.

\section{3. Задача оптимизации}

Чтобы облегчить эффективную поддержку качества обслуживания $(\mathrm{QoS})$ в беспроводных сетях следующего поколения, важно смоделировать беспроводной канал с точки зрения таких показателей QoS на уровне соединения, как скорость передачи данных, задержка и вероятность нарушения задержки. В то же время нельзя забывать и об уровне предоставления услуги (SLA - Service Level Agreement). К ключевым понятиям можно отнести: качество, доступность, обязанности. Эти дисциплины согласовываются между поставщиком и пользователем используемой услуги [5].

В связи с этим необходимо провести максимизацию средней скорости трафика еMBВ в описанной выше модели. Получается, что параметр $b^{*}$, описываемый как значение, ниже которого не должна опускаться скорость заявок, не должен быть ниже начального $b_{K}$. Также важно учесть тот факт, что средняя скорость зависит и от начальных скоростных значений, т.е. $W=\left\{w_{1}, \ldots, w_{B}\right\}$ и от количества $K$. По определению имеем начальное условие: $b_{1}>b_{2}>\ldots>b_{K}$. Следовательно, описать задачу оптимизации средней скорости можно следующим образом:

$$
\begin{gathered}
\bar{b}\left(K, b_{1}, \ldots, b_{K}\right) \rightarrow \max \\
\left\{\begin{array}{l}
b_{K} \in W\left\{w_{1}, \ldots, w_{B}\right\}, k=\overline{1, K}, \\
b_{1}>b_{2}>\ldots>b_{K}, \\
b_{K} \geq b^{*} .
\end{array}\right.
\end{gathered}
$$

\section{Заключение}

В работе рассмотрена модель совместного обслуживания двух видов трафика со снижением скорости и прерыванием обслуживания трафика еМВВ. Представлены условия для распределения вероятностей в матричном виде, выведены формулы вероятностно-временных характеристик. Сформулирована задача максимизации средней скорости.

\section{ЛИТЕРАТУРА}

1. Ishan Budhiraja, Sudhanshu Tyagi, Sudeep Tanwar, Neeraj Kumar, Joel J.P.C. Rodrigues Tactile Internet for Smart Communities in 5G: An Insight for NOMA-based Solutions. - 2019.

2. 3GPP, 'Technical Specification Group Radio Access Network; Study on Scenarios and Requirements for Next Generation Access Technologies;', TR 38.913 Release 14, V.14.3.0, 2017.

3. Makeeva E., Polyakov N., Kharin P., Gudkova I. Probability Model for Performance Analysis of Joint URLLC and eMBB Transmission in 5G Networks. // Internet of Things, Smart Spaces, and Next Generation Networks and Systems. NEW2AN 2019, ruSMART 2019. Lecture Notes in Computer Science. - 2019. - V. 11660. - Springer, Cham. https://doi.org/10.1007/978-3-030-30859-9 55.

4. Башарин Г.П. Лекции по математической теории телетрафика. - М.: РУДН, 2004.

5. Yan Chen, Toni Farley, Nong Ye QoS Requirements of Network Applications on the Internet // Information Knowledge Systems Management 4. - 2004. - P. 55-76. 